\title{
The edge-count criterion for graphic lists
}

\author{
Garth Isaak \\ Department of Mathematics \\ Lehigh University \\ Bethlehem, PA 18015, U.S.A. \\ gisaak@lehigh.edu
}

\author{
Douglas B. West* \\ Mathematics Department \\ University of Illinois \\ Urbana IL, U.S.A. \\ west@math.uiuc.edu
}

\begin{abstract}
Submitted: Sep 14, 2009; Accepted: Jan 28, 2010; Published: Nov 19, 2010
Mathematics Subject Classification: $05 \mathrm{C} 07$
\end{abstract}

\begin{abstract}
We give a new short proof of Koren's characterization of graphic lists, extended to multigraphs with bounded multiplicity $p$, called $p$-graphs. The Edge-Count Criterion (ECC) for an integer $n$-tuple $d$ and integer $p$ is the statement that for all disjoint sets $I$ and $J$ of indices, $\sum_{i \in I} d_{i}+\sum_{j \in J}\left[p(n-1)-d_{j}\right] \geqslant p|I||J|$. An integer list $d$ is the degree list of a $p$-graph if and only if it has even sum and satisfies ECC. Analogous statements hold for bipartite or directed graphs, and an old characterization of degree lists of signed graphs follows as a corollary of the extension to multigraphs.
\end{abstract}

The problem of characterizing degree lists (also called "degree sequences") of simple graphs is well studied. The sum is twice the number of edges and hence must be even, but this condition is not sufficient. Sierksma and Hoogeveen [11] summarized seven characterizations. With additional results, these also appear in [7]. The various characterizations have been proved in many ways; we will not attempt to survey the proofs.

We give a new short proof of another natural characterization, due to Koren [6], which we call the Edge-Count Criterion. Koren used it to characterize the polytope of degree lists [10]. We prove the characterization in the more general setting of multigraphs with bounded multiplicity $p$. The idea also works for bipartite or directed graphs, and the multigraph characterization applies to give an immediate characterization of degree lists for signed graphs (using a transformation due to T.S. Michael).

A multigraph $G$ with bounded multiplicity $p$ is a pair consisting of a set $V(G)$ of vertices and a multiset $E(G)$ of unordered pairs of vertices, where each pair occurs at most $p$ times as an edge. Motivated by Berge [1], we call such a multigraph a $p$-graph (the 1-graphs are the graphs or simple graphs). Let $\mu(x y)$ denote the multiplicity of an edge

\footnotetext{
*Research partially supported by the National Security Agency under Award No. H98230-10-1-0363.
} 
$x y$; if $\mu(x y)>0$, then $x$ and $y$ are adjacent. The complement of a $p$-graph $G$, denoted $\bar{G}$, is the $p$-graph with vertex set $V(G)$ such that $\mu_{\bar{G}}(x y)=p-\mu_{G}(x y)$ for all $x y \in\left(\begin{array}{c}V(G) \\ 2\end{array}\right)$. The degree of a vertex $v$, written $d(v)$, is the sum of the multiplicities of the pairs containing $v$. We write an integer list $\left(d_{1}, \ldots, d_{n}\right)$ simply as $d$. An integer $n$-tuple $d$ is p-graphic if the entries are the vertex degrees of some $p$-graph. Such a $p$-graph is a realization of $d$. Let $[n]=\{1, \ldots, n\}$.

Definition 1. An integer $n$-tuple d satisfies the Edge-Count Criterion (ECC) for $p$-graphs if for all $I, J \subseteq[n]$ with $I \cap J=\varnothing$,

$$
\sum_{i \in I} d_{i}+\sum_{j \in J}\left[p(n-1)-d_{j}\right] \geqslant p|I||J|
$$

We call this the Edge-Count Criterion because always $\mu_{G}(x y)+\mu_{\bar{G}}(x y)=p$. The sum on the left counts degrees in $G$ for vertices of $I$ and in $\bar{G}$ for vertices of $J$. The total must account for the total multiplicity of all pairs in $I \times J$, regardless of how it splits between $G$ and $\bar{G}$. Thus the condition is necessary. We will give a short proof that when even sum is also required it becomes sufficient.

Koren's statement of the ECC for 1-graphs, when expressed in our notation, was $\sum_{j \in J} d_{j} \leqslant \sum_{i \in I} d_{i}+|J|(n-1-|I|)$. We have reordered the terms to facilitate a short proof and express the natural generalization to $p$-graphs. Characterizations of $p$-graphic lists were given by Chungphaisan [2] and by Berge [1].

Fulkerson-Hoffman-McAndrew [5] proved that every 1-graphic list has a realization in which any specified vertex $v$ is adjacent to vertices whose degrees are the largest entries in the list other than its own. We need the extension to $p$-graphs of an easy special case.

Lemma 2. Let $d$ be a p-graphic list with largest entry $k$. If $d_{j}>0$ and $d_{j}$ is not the only $k$ in $d$, then in some realization a vertex of degree $d_{j}$ is adjacent to a vertex of degree $k$.

Proof. Let $G$ be a realization of $d$. Let $x$ and $v$ be vertices of degrees $k$ and $d_{j}$. If $\mu(x v)=0$, then $v$ is adjacent to some other vertex $u$. Since $d(u) \leqslant k$, and $v$ is adjacent to $u$ but not $x$, there exists $y$ such that $\mu(x y)>\mu(u y)$. Decreasing $\mu(x y)$ and $\mu(v u)$ by 1 and increasing $\mu(x v)$ and $\mu(u y)$ by 1 yields a realization as desired.

Theorem 3. An integer n-tuple $d$ with even sum is p-graphic if and only if it satisfies the ECC for p-graphs.

Proof. We have observed that the conditions are necessary. For sufficiency, we use induction on $n+\sum d_{i}$. For a list $d$, let $S_{I, J}(d)$ denote $\sum_{i \in I} d_{i}+\sum_{j \in J}\left[p(n-1)-d_{j}\right]$, so ECC states that $S_{I, J}(d) \geqslant p|I||J|$ whenever $I$ and $J$ are disjoint.

Suppose that $d$ satisfies ECC. Using pairs $I, J$ in which one set is empty and the other has size 1 , we obtain $0 \leqslant d_{i} \leqslant p(n-1)$ for all $i$, so the induction parameter is positive. When it equals 1 , the only realization is the unique 1-vertex $p$-graph, which has no edges.

For the induction step, index $d$ so that $d_{1}$ is a largest entry and $d_{n}$ is smallest. If $d_{n}=0$, then form $d^{\prime}$ by deleting $d_{n}$. Since $d^{\prime}$ is an $(n-1)$-tuple and ECC holds for $d$, we have $S_{I, J}\left(d^{\prime}\right)=S_{I \cup\{n\}, J}(d)-p|J| \geqslant p(|I|+1)|J|-p|J|=p|I||J|$. Thus ECC holds for 
$d^{\prime}$, which has the same sum as $d$. By the induction hypothesis $d^{\prime}$ is $p$-graphic, and adding an isolated vertex to a realization of $d^{\prime}$ yields a realization of $d$.

Hence we may assume $d_{n} \geqslant 1$. Form $d^{\prime}$ by subtracting 1 from the first and last entries. If $d^{\prime}$ is $p$-graphic, then applying Lemma 2 to the complement of a realization of $d^{\prime}$ yields a realization of $d^{\prime}$ having vertices $x$ and $y$ of degrees $d_{1}-1$ and $d_{n}-1$ such that $\mu(x y)<p$. Increasing the multiplicity of $x y$ completes a realization of $d$.

Since $d^{\prime}$ has even sum, by the induction hypothesis it suffices to show that $d^{\prime}$ satisfies ECC. If $d_{i}^{\prime}>d_{j}^{\prime}$ for some $i \in I$ and $j \in J$, then moving $i$ to $J$ and $j$ to $I$ reduces $S_{I, J}\left(d^{\prime}\right)$ without changing $|I||J|$. Hence it suffices to prove $(*)$ when $d_{i}^{\prime} \leqslant d_{j}^{\prime}$ for $i \in I$ and $j \in J$.

Writing $(*)$ as $\sum_{i \in I}\left(d_{i}^{\prime}-p|J|\right)+\sum_{j \in J}\left[p(n-1)-d_{j}^{\prime}\right] \geqslant 0$, we need only prove $(*)$ when $d_{i}^{\prime}<p|J|$ for $i \in I$. Furthermore, if $d_{i^{\prime}}^{\prime}<d_{i}^{\prime}<p|J|$ and $i \in I$, then $i^{\prime} \notin J$, and adding $i^{\prime}$ to $I$ if not already in $I$ reduces the left side. Hence we may assume that all entries smaller than any indexed by $I$ are also indexed by $I$. Similarly, to ensure $\sum_{i \in I} d_{i}^{\prime}+\sum_{j \in J}\left[p(n-1-|I|)-d_{j}^{\prime}\right] \geqslant 0$, we may assume that $d_{j}^{\prime}>p(n-1-|I|)$ for $j \in J$, and entries larger than any indexed by $J$ are indexed by $J$.

Since $0 \leqslant d_{i}^{\prime} \leqslant p(n-1),(*)$ holds when $I$ or $J$ is empty. Hence we may assume that both are nonempty, with $J$ containing the index of a largest entry and $I$ containing that of a smallest. In particular, $n \in I$. If $d_{j}^{\prime}=d_{1}-1$ for any $j \in J$ (including $j=1$ ), then $S_{I, J}\left(d^{\prime}\right)=S_{I, J-\{j\}+\{1\}}(d) \geqslant|I||J|$. Hence we may assume that $d_{j}^{\prime}=d_{1}$ for $j \in J$.

For $j \in J$, we have $d_{1}=d_{j}^{\prime}>p(n-1-|I|)$, or $-p(n-1)-d_{1}>-p|I|$. If $1 \notin I$, then

$$
S_{I, J}\left(d^{\prime}\right)=S_{I, J \cup\{1\}}(d)-1-\left[p(n-1)-d_{1}\right] \geqslant p|I|(|J|+1)-p|I|=|I||J| .
$$

Hence we may assume $1 \in I$. Now $d_{1}^{\prime}<p|J|$, so $d_{1} \leqslant p|J|$. With $|I|+|J| \leqslant n$,

$$
\begin{aligned}
S_{I, J}\left(d^{\prime}\right) & =\left(\sum_{i \in I} d_{i}\right)-2+\left[p(n-1)-d_{1}\right]|J| \geqslant d_{1}+|I|-1-2+\left[p(|I|+|J|-1)-d_{1}\right]|J| \\
& =|I|-3+p|I||J|+(|J|-1)\left(p|J|-d_{1}\right) .
\end{aligned}
$$

Failure requires $(|J|-1)\left(p|J|-d_{1}\right)<3-|I|$ and equality throughout the computation. Hence $I=\{1, n\}$ and $|J| \in\left\{1, d_{1} / p\right\}$; also $d_{n}=1$ and $|I|+|J|=n$, so $|J|=n-2$. If $|J|=1$, then $d=\left(d_{1}, d_{1}, 1\right)$. If $p|J|=d_{1}$, then $d=(p(n-2), \ldots, p(n-2), 1)$, with $n-1$ entries equal to $p(n-2)$. In each case, $d$ has odd sum, so these possibilities are excluded.

Hence $d^{\prime}$ satisfies ECC, and the induction hypothesis applies to complete the proof.

When $p=1$, some cases disappear earlier. The requirement for $d_{i}=d_{j}$ with $i \in I$ and $j \in J$ is $p|J|>d_{i}^{\prime}=d_{j}^{\prime}>p(n-1-|I|)$, which simplifies to $|I|+|J| \geqslant n-1+2 / p$ and cannot hold when $p=1$. Therefore, when $p=1$ we may assume that $I=\left\{i: d_{i}^{\prime}<|J|\right\}$ and $J=\left\{j: d_{j}^{\prime}>n-1-|I|\right\}$. This leads more quickly to $n \in I$ and $d_{1}=n-|I|$.

For bipartite graphs there is a similar characterization. A pair of lists $\left(r_{1}, \ldots, r_{m}\right)$ and $\left(s_{1}, \ldots, s_{n}\right)$ is bigraphic if there is a bipartite graph with partite sets $X$ and $Y$ such that $r$ is the list of degrees of vertices in $X$ and $s$ is the list of degrees of vertices in $Y$. As above, we consider bipartite $p$-graphs. A characterization follows from a more general result of Ore [9], which we state in our notation: A bipartite graph $G$ with partite sets $[m]$ and $[n]$ 
has a spanning subgraph with degree lists $r$ for $[m]$ and $s$ for $[n]$ if and only if, whenever $I \subseteq[m]$ and $J \subseteq[n], \sum_{i \in J} s_{j}$ is at most $\sum_{i \in I} r_{i}$ plus the number of edges joining $J$ and $[m]-I$. When $G$ is a complete bipartite $p$-graph, this reduces to the following statement.

Theorem 4. Integer lists $r$ and $s$ form the degree lists for a bipartite p-graph if and only if they satisfy the Bipartite Edge-Count Criterion that for all $I \subseteq[m]$ and $J \subseteq[n]$,

$$
\sum_{i \in I} r_{i}+\sum_{j \in J}\left(p m-s_{j}\right) \geqslant p|I||J|
$$

For $p=1$, this is known as the Gale-Ryser Theorem. It can be proved using network flow methods or by a short inductive proof. A proof parallel to that of Theorem 3 is also quite short, since the difficult case $(1 \in I)$ does not occur in the bipartite setting. We omit the analogous statement for directed graphs.

The ECC also applies to characterize degree lists of "signed" p-graphs. In a signed multigraph, each edge is positive or negative, and the degree of a vertex is the number of incident positive edges minus the number of incident negative edges (loops contribute twice at their vertex). For signed $p$-graphs, we forbid loops, and each vertex pair has multiplicity at most $p$ as a positive edge and as a negative edge. Since copies of a single edge with opposite sign contribute 0 to the degree of its endpoints, for purposes of realizability we may view a signed $p$-graph as an edge-weighted complete graph with integer weights in the interval $[-p, p]$.

T.S. Michael [8] observed that signed $p$-graphs without repeated edges having opposite sign are equivalent to unsigned $2 p$-graphs. The correspondence is simply to add $p$ to each edge weight in the interpretation as a weighted complete graph. This adds $p(n-1)$ to each degree. Michael then observed characterizations of signed $p$-graphs using characterizations of $2 p$-graphs, but the particularly simple consequence of the ECC was not included. The result, observed by Kyle Jao (private communication), is

Theorem 5. An integer $n$-tuple $d$ is the degree list of a signed p-graph if and only if all disjoint $I, J \subseteq[n]$ satisfy

$$
\sum_{i \in I}\left[p(n-1)+d_{i}\right]+\sum_{j \in J} d_{j} \geqslant 2 p|I||J| .
$$

\section{References}

[1] Berge, C., Graphes et Hypergraphe, Dunod, Paris, 1970.

[2] Chungphaisan, V., Conditions for sequences to be $r$-graphic, Discrete Math., 7 (1974), 31-39.

[3] Erdos, P. and Gallai, T., Graphs with prescribed degrees of vertices (Hungarian), Mat. Lapok, 11 (1960), 264-274.

[4] Ford, L.R. and Fulkerson, D.R., Flows in Networks, Princeton Univ. Press, 1962. 
[5] Fulkerson, D.R., Hoffman, A.J. and McAndrew, M.H., Some properties of graphs with multiple edges, Canad. J. Math., 17 (1965), 166-177.

[6] Koren, M., Extreme degree sequences of simple graphs, J. Combin. Theory B, 15 (1973), 213-224.

[7] Mahadev, N. V. R. and Peled, U. N., Threshold graphs and related topics. Annals of Discrete Mathematics, 56. North-Holland Publishing Co., Amsterdam, 1995.

[8] Michael, T.S., Signed degree sequences and multigraphs, J. Graph Theory 41 (2002), 101-105.

[9] Ore, O., Studies in directed graphs I, Ann. of Math. (2) 63 (1956), 383-406.

[10] Peled, U.N. and Srinivasan, M.K., The polytope of degree sequences, Linear Algebra Appl. 114/115 (1989) 349-377.

[11] Sierksma, G. and Hoogeveen, H., Seven criteria for integer sequences being graphic, J. Graph Theory 15 (1991), 223-231. 